\title{
The global prevalence of Wilson disease from next-generation sequencing data
}

\author{
Jiali Gao, BA (Hons) ${ }^{1}$, Simon Brackley, BA (Hons) ${ }^{1}$ and Jake P. Mann, MRCP MRCPCH ${ }^{2,3}$
}

Purpose: Wilson disease (WD) is an autosomal recessive disorder of copper metabolism, caused by pathogenic variants in ATP7B. We aimed to (1) perform a meta-analysis of previous WD prevalence estimates, (2) estimate the prevalence of WD from population sequencing data, and (3) generate an $A T P 7 B$ gene variant database.

Methods: MEDLINE and EMBASE were systematically searched. Previous prevalence estimates were subjected to meta-analysis. All previously reported pathogenic $A T P 7 B$ variants were compiled and annotated with gnomAD allele frequencies. Pooled global and ethnicity-specific genetic prevalences for WD were generated using the Hardy-Weinberg equation.

Results: Meta-analysis of genetic studies of WD prevalence gave an estimate 12.7 per 100,000 (95\% confidence interval [CI]: 6.3-23.0). We developed a referenced, searchable $A T P 7 B$ database comprising 11,520 variants including 782 previously reported disease variants, which can be found at http://www.wilsondisease. $\mathrm{tk} / ; 216 / 782$ of these were present in gnomAD, remained after filtering by allele frequency, and met American College of Medical Genetics and Genomics criteria. Based on these, the genetic prevalence of WD was 13.9 per 100,000 (95\% CI: 12.9-14.9), or 1 per 7194. Combining this with 60 predicted pathogenic variants gave a birth prevalence of 15.4 per 100,000 (95\% CI: 14.4-16.5).

Conclusion: The genetic prevalence of Wilson disease may be greater than previous estimates.

Genetics in Medicine (2019) 21:1155-1163; https://doi.org/10.1038/s41436018-0309-9

Keywords: Wilson disease; $A T P 7 B$; prevalence; pathogenic variants; database

\section{INTRODUCTION}

Wilson disease (WD) is an autosomal recessive disorder of copper metabolism (OMIM 277900), in which there is defective transport of copper across the endoplasmic reticulum and biliary copper excretion. This manifests as hepatic, neurological, and psychiatric symptoms. Diagnosis is based on a combination of clinical features, serum ceruloplasmin, urinary copper and hepatic copper, ${ }^{1}$ with early detection and effective treatment resulting in a normal lifespan with minimal morbidity.

WD is known to be caused by pathogenic variants (PV) in the $A T P 7 B$ gene. Although the possibility of a second WD gene has been discussed, no other genes have been identified. $^{2}$ Indeed, study sequencing 181 patients in the United Kingdom found an overall $A T P 7 B$ pathogenic variant detection frequency of $98 \%$ (ref. ${ }^{3}$ ), supporting the belief that WD is a classic monogenic disorder. However, the mutational spectrum is wide, with the WD Mutation Database by the University of Alberta (last updated in 2010) listing around 500 pathogenic variants ${ }^{4}$ though the actual number may be greater. $A T P 7 B$ pathogenic variant testing may be required for the diagnosis of WD but it is not yet routine. ${ }^{1}$

The prevalence of WD is often quoted as 1 in 30,000, taken from a monograph written by Scheinberg and Sternlieb in 1984, before the discovery of the gene responsible. ${ }^{5}$ This estimate was based on three studies that have been described as methodologically flawed ${ }^{6}$ and it has been recognized some regions have a much higher prevalence. In accordance with this, recent estimates from sequencing studies have been much higher, with several papers raising the possibility of underdiagnosis., ${ }^{3,7,8}$ Moreover, reports of WD in consecutive generations of families, producing a "pseudodominant" inheritance pattern, suggest that heterozygous $A T P 7 B$ pathogenic variant carriers may be more common than previously thought. ${ }^{3}$

Therefore, we aimed to, firstly, perform a meta-analysis of previous prevalence estimates for WD and secondly, estimate the prevalence of WD across ethnicities from publicly available population sequencing data, using a validated methodology. ${ }^{9}$ In addition, we aimed to compile

\footnotetext{
${ }^{1}$ University of Cambridge, School of Clinical Medicine, Cambridge, UK; ${ }^{2}$ Department of Paediatrics, University of Cambridge, Cambridge, UK; ${ }^{3}$ Institute of Metabolic ScienceMetabolic Research Laboratories, University of Cambridge, Cambridge, UK. Correspondence: Jake P. Mann (jm2032@cam.ac.uk)

These authors contributed equally: Jiali Gao and Simon Brackley.
}

Submitted 29 May 2018; accepted: 5 September 2018

Published online: 26 September 2018 
a new database of reported WD pathogenic variants by systematically searching the literature and cross-referencing with pre-existing databases.

\section{MATERIALS AND METHODS}

The protocol for this study was registered with PROSPERO (https://www.crd.york.ac.uk/PROSPERO/display_record.php? RecordID $=74489$ )

\section{Meta-analysis of previous prevalence estimates}

Both MEDLINE and EMBASE were systematically searched for papers related to Wilson disease prevalence or epidemiology on 15 August 2017 (see supplementary data for search terms). Two independent reviewers (J.G. and S.B.) screened abstracts to determine suitability for inclusion. Any disagreement was resolved through discussion with a third reviewer (J. M.). Foreign language reports; reviews, commentaries, and editorials reporting nonoriginal data; and in vitro or nonhuman studies were excluded; papers quoting an original estimate of WD prevalence or incidence were included.

Included papers were quality reviewed and assessed for risk of bias using the Appraisal tool for Cross-Sectional Studies (AXIS) ${ }^{10}$ (Figure S3) and prevalence data were extracted. Where appropriate, prevalence was estimated from the number of cases reported in the paper and relevant population size data obtained from official statistics, as specified in the results. In addition, where multiple prevalence estimates over time were given, the most recent figure was taken.

Studies were subclassified into epidemiological studies, defined as those based on case frequencies within a population, and genetic studies, involving sequencing for WD pathogenic variants and calculation of mutant allele frequencies.

The degree of study heterogeneity was investigated by means of the Cochran Q test and its related metric $\mathrm{I}^{2}$. Because in all cases there was significant heterogeneity, estimates of prevalence from epidemiological studies and mutant allele frequencies from genetic studies were subjected to metaanalysis separately using random effects models. ${ }^{11}$ Population size was taken to be the overall population of the catchment area for epidemiological studies and sample size for genetic studies. Where population size was unclear in the paper, population data for the appropriate year was obtained from the World Bank (https://data.worldbank.org, accessed 14 March 2018), unless otherwise specified. From the overall mutant allele frequency an estimate of the prevalence of WD at birth was calculated using the Hardy-Weinberg equation.

All statistical analysis was performed using the Meta-XL add-in for Microsoft Excel (www.epigear.com). Forest plots were generated using DistillerSR Forest Plot Generator from Evidence Partners (www.evidencepartners.com)

\section{Identification of reported pathogenic variants and functional variants}

A systematic search was performed to produce a list of all known disease-causing pathogenic variants in the $A T P 7 B$ gene. Both MEDLINE and EMBASE were searched for papers relating to WD pathogenic variants or genetics on 15 August 2017 (see supplementary data for search terms). As above, two independent reviewers screened abstracts to determine suitability for inclusion and disagreements were resolved through discussion with a third reviewer. Foreign language reports; reviews, commentaries, and editorials reporting nonoriginal data; and in vitro or nonhuman studies were excluded; papers reporting variants within the $A T P 7 B$ gene were included. Variants reported as disease-causing were extracted.

The University of Alberta WD database ${ }^{12}$ (www.wilsondisease. med.ualberta.ca/references.asp, accessed 2 March 2018), ClinVar (www.ncbi.nlm.nih.gov/clinvar/, accessed 13 November 2017) were screened for additional $A T P 7 B$ variants with published reports of pathogenicity and these were added to our list. Variants derived from personal communications and unpublished data were excluded. Compound pathogenic variants, defined as more than one nonoverlapping mutation within the same $A T P 7 B$ sequence, were also excluded.

A list of all known variants in $A T P 7 B$, including polymorphisms, was compiled. All above variants were combined with variants from the University of Alberta WD database and ClinVar without evidence of pathogenicity and additional variants from Ensembl (www.ensembl.org, accessed 26 January 2018) and gnomAD (gnomad. broadinstitute.org, accessed 26 January 2018).

\section{Annotation of variants with allele frequencies and functional predictions}

Coding sequence nucleotide changes for each variant were identified and converted to Human Genome Variation Society (HGVS) format (hg38) using Mutalyzer (https:// mutalyzer.nl/). The Ensembl Variant Effect Predictor (https:// ensembl.org/Tools/VEP) was used to annotate variants with mutation consequences and SIFT/PolyPhen in silico predictions of pathogenicity. GnomAD allele frequency data was downloaded directly and added to each variant.

\section{Frequency filtering}

All variants with allele frequency data available from the gnomAD data set were filtered using a method proposed by Whiffin et al. ${ }^{13} \mathrm{~A}$ "maximum credible population allele frequency" was calculated based on the equation:

$$
\begin{gathered}
\text { Max credible population } A F= \\
\sqrt{\text { prevalence }} \times \text { max allelic contribution } \\
\times \sqrt{\text { max genetic contribution }} \times \frac{1}{\sqrt{\text { penetrance }}}
\end{gathered}
$$

Reliable estimates for these parameters were difficult to obtain, so an upper bound for the maximum credible allele frequency (AF) was calculated. Prevalence was taken from the meta-analysis estimate from genetic studies, 1 in 7874 , as these studies are most comparable with our current method. Maximum allelic contribution, the maximum proportion of variation within a gene attributable to a single allele, was set at $30 \%$, based on a variety of estimates 
Table 1 Epidemiological studies estimating WD prevalence

\begin{tabular}{|c|c|c|c|c|c|c|}
\hline Paper & Location & Age range & Year & $\begin{array}{l}\text { Population } \\
\text { size (1000 s) }\end{array}$ & $\begin{array}{l}\text { Prevalence } \\
\text { (per 100,000) }\end{array}$ & AXIS assessment \\
\hline Poujois et al. ${ }^{19}$ & France & All & 2013 & 58,000 & 1.50 & $\begin{array}{l}\text { High quality, very low Risk } \\
\text { of Bias (RoB) }\end{array}$ \\
\hline Tai et al. ${ }^{20}$ & Taiwan & All & $2000-2011$ & $23,162^{b}$ & 1.81 & High quality, very low RoB \\
\hline Lai et al. ${ }^{21, a}$ & Taiwan & All & 2005 & 22,770 & 1.60 & High quality, very low RoB \\
\hline Cheng et al. ${ }^{22}$ & Anhui, China & $7-75$ & $2008-2011$ & 2700 & 5.87 & $\begin{array}{l}\text { Medium quality, risk of } \\
\text { sampling bias }\end{array}$ \\
\hline Moller et al. ${ }^{23}$ & Denmark & All & $1990-2008$ & 5494 & 2.02 & High quality, very low RoB \\
\hline Giagheddu et al. ${ }^{24}$ & Sardinia & All & Unclear & 74 & 2.77 & High quality, low RoB \\
\hline Adhami et al. ${ }^{25}$ & Albania & All & $?-1991$ & 3267 & 0.68 & $\begin{array}{l}\text { Low quality, unclear RoB, } \\
\text { unclear methods }\end{array}$ \\
\hline Reilly et al. ${ }^{26}$ & Ireland & All & 1986 & $3541^{c}$ & 0.54 & High quality, low RoB \\
\hline Park et al. ${ }^{6}$ & Scotland & All & 1989 & 5091 & 0.40 & High quality, low RoB \\
\hline Bonne-Tamir et al. ${ }^{27}$ & Israel & All & 1958-1985 & 4106 & $0.25^{d}$ & High quality, low RoB \\
\hline Garcia-Villarreal et al. ${ }^{28, a}$ & $\begin{array}{l}\text { NE Canary } \\
\text { Islands, Spain }\end{array}$ & All & 1981 & 1586 & 38.50 & $\begin{array}{l}\text { Medium quality, risk of } \\
\text { nonresponder bias }\end{array}$ \\
\hline Dedoussis et al. ${ }^{29, a}$ & Cretan village & All & 1978-? & Unclear & 6666.67 & High quality, low RoB \\
\hline
\end{tabular}

AXIS Appraisal tool for Cross-Sectional Studies, ${ }^{10}$ WD Wilson disease.

${ }^{a}$ Excluded from further analysis.

bSource: www.ndc.gov.tw, accessed 14 March 2018.

cwww.cso.ie, accessed 14 March 2018.

dEstimated from number of cases in study population detailed in paper.

of p.His1069Gln $A T P 7 B$ variant prevalence in WD patients. ${ }^{3,8,14,15}$ Maximum genetic contribution, the maximum proportion of disease attributable to variation within a gene, was set at $98 \%$ based on Coffey et al. ${ }^{3}$ Lastly, the penetrance used for this calculation was selected as $50 \%$, as suggested by the original authors' methods. This gave a maximum credible AF of $0.473 \%$.

Filtering allele frequencies were also computed for each variant based on gnom $A D$ allele counts, using the $\mathrm{R}$ code provided by Whiffin et al. Variants with filtering AFs greater than the maximum credible AF were excluded from further analysis.

\section{Disease variant classification}

Variants reported as pathogenic with allelic frequency data were further screened and classified using American College of Medical Genetics and Genomics (ACMG) criteria. ${ }^{16}$ Variants reported in a peer-reviewed journal were labeled with PS4, PP4, and PP5 level evidence and classified as "likely pathogenic" if they:

1. Were associated with at least two of the following criteria: ${ }^{5}$

- Low ceruloplasmin level $<20 \mathrm{mg} / \mathrm{dl}$

- The presence of Kayser-Fleischer rings by slit-lamp examination

- Hepatic copper content of $250 \mathrm{mcg} / \mathrm{g}$ dry weight liver tissue

- 24 hour urinary copper $>100 \mathrm{mcg}$
In the presence of hepatic or neurological manifestations consistent with WD:

2. Had a significantly increased prevalence in affected individuals compared with controls

\section{Prevalence estimation}

Allele frequencies of relevant variants were extracted from our $A T P 7 B$ variant database, pooled, and estimates of the prevalence of WD at birth were generated using the Hardy-Weinberg equation.

Ninety-five percent confidence intervals (CIs) for these estimates were calculated as Wilson interval scores by the Wilson score method. Graphs were generated using Microsoft Excel (2016) for Windows.

\section{RESULTS}

\section{Previous estimates of prevalence}

One thousand three abstracts were identified as potentially eligible, of which 20 studies were included; $12 / 20$ (60\%) of these employed an epidemiological method and 8/20 (40\%) used genetic sequencing (Figure S1).

From these, 22 estimates of prevalence were extracted, from various locations worldwide and reporting population prevalences ranging from 0.25 to $6667 / 100,000$ (Table 1 and Table 2). Three epidemiological studies were excluded from the meta-analysis: Dedoussis et al. (2005) and Garcia-Villarreal et al. (2000) describe outlier populations; and Lai et al. (2010) due to geographical and temporal overlap with Tai et al. (2017). Genetic studies with geographical 
Table 2 Genetic studies estimating WD prevalence at birth

\begin{tabular}{|c|c|c|c|c|c|}
\hline Paper & Method & Location & $\begin{array}{l}\text { Sample } \\
\text { size }\end{array}$ & $\begin{array}{l}\text { Birth prevalence } \\
\text { (per 100,000) }\end{array}$ & AXIS assessment \\
\hline Jang et al. ${ }^{7}$ & $\begin{array}{l}\text { DNA analysis of neonatal DBS for } \\
6 \text { pathogenic variants }\end{array}$ & Korea & 14,835 & 13.23 & High quality, very low RoB \\
\hline Jang et al. ${ }^{7}$ & $\begin{array}{l}\text { Retrospective review of sequencing } \\
\text { data for any WD disease variant }\end{array}$ & Korea & 1090 & 9.22 & High quality, very low RoB \\
\hline Kim at al. ${ }^{30}$ & $\begin{array}{l}\text { DNA analysis of neonatal DBS for } \\
3 \text { pathogenic variants }\end{array}$ & Korea & 476 & 3.05 & High quality, very low RoB \\
\hline Coffey et al. ${ }^{3}$ & $\begin{array}{l}\text { DNA analysis of neonatal DBS for } \\
\text { any WD disease variant }\end{array}$ & United Kingdom & 1000 & 14.23 & High quality, very low RoB \\
\hline Zappu et al. ${ }^{31}$ & $\begin{array}{l}\text { DNA analysis of neonates for } \\
2 \text { pathogenic variants }\end{array}$ & Kalymnos, Greece & 397 & 13.50 & High quality, very low RoB \\
\hline Zappu et al. ${ }^{31}$ & $\begin{array}{l}\text { DNA analysis of neonates for } \\
1 \text { pathogenic variant }\end{array}$ & Sardinia & 5290 & 35.75 & High quality, very low RoB \\
\hline Krumina et al. ${ }^{15}$ & $\begin{array}{l}\text { DNA analysis of healthy controls } \\
\text { for } 1 \text { pathogenic variant }\end{array}$ & Latvia & 157 & 3.91 & High quality, low RoB \\
\hline Olivarez et al. ${ }^{8}$ & $\begin{array}{l}\text { DNA analysis of neonates for } \\
1 \text { pathogenic variant }\end{array}$ & $\begin{array}{l}\text { New York state, } \\
\text { United States }\end{array}$ & 2456 & 1.82 & High quality, very low RoB \\
\hline
\end{tabular}

Jang et al. ${ }^{7}$ and Zappu et al. ${ }^{31}$ are represented twice as they each report two separate estimates based on different populations.

AXIS Appraisal tool for Cross-Sectional Studies, DBS dried blood spot, WD Wilson disease.

overlap were not excluded since each study only sampled a small proportion of the target population, so overlap in study cohort was deemed unlikely.

Meta-analysis of epidemiological estimates of prevalence using the inverse variance method with a double arcsin transformation gave a pooled prevalence of 1.38 (95\% CI: $0.85-2.05$ ) per 100,000 (Fig. 1a). Meanwhile, meta-analysis of mutant allele frequencies estimates from genetic sequencing studies gave a pooled allele frequency of 0.011 (95\% CI: 0.008-0.015) (Fig. 1b). Using the Hardy-Weinberg equation, this mutant allele frequency is equivalent to a prevalence at birth of 12.7 per 100,000 (95\% CI: 6.25-23.0). The studies in both meta-analyses showed statistically significant heterogeneity (epidemiological: $I^{2}=98.0 \%, p<0.001$; genetic: $I^{2}=$ $76.9 \%, p<0.001)$.

We then proceeded to attempt to obtain a more reliable estimate of global WD prevalence, using publicly available sequencing data.

\section{Identification of ATP7B Variants}

Our systematic search for WD pathogenic variants returned 1558 abstracts, of which 245 papers were included, from which 771 pathogenic variants were extracted (Figure S2). Screening of the University of Alberta WD database and of ClinVar for variants reported as disease-causing in the published literature found an additional 10 variants and 1 variant, respectively, giving a total of 782 reported disease variants (Fig. 2a). GnomAD allele frequency data was available for $238 / 782$ (30.4\%) of these disease-causing variants.
A list of all known variants in $A T P 7 B$, including nonpathogenic variants and polymorphisms, was also compiled. The above-reported disease variants were combined with 10,199 variants from Ensembl, 2301 from gnomAD, 372 from ClinVar, and 654 from the University of Alberta WD database, giving a total of 11,520 variants (Fig. 2a). The full database can be found at http://www.wilsondisease.tk/ and in Table S2. GnomAD allele frequency data were available for $2301 / 11,520(19.9 \%)$ of these variants.

\section{Analysis of allele frequencies Known pathogenic variants}

Of 238 reported disease-causing variants with allele frequency data, 234 remained after frequency filtering and 216 of these were classified as "likely pathogenic" under ACMG criteria (Fig. 2a). Pooling of the allele frequencies of these variants gave a global mutant allele frequency of 0.0118 , which is equivalent to a prevalence at birth of 13.9 per 100,000 (95\% CI: 12.9-14.9), or 1 per 7194 (Fig. 2b and Table S1). The East Asian ethnicity had the highest estimated prevalence of 29.5 per 100,000 (95\% CI: 23.6-36.8) compared with only 2.08 per 100,000 (95\% CI: $1.43-3.03$ ) in the Finnish population. There was insufficient variant-level penetrance data to adjust our estimate based on the cumulative penetrance of disease variants.

The most common mutation consequence was missense, accounting for $52 \%$ of all likely pathogenic variants (including those without allele frequency data) and contributing $85 \%$ of the total mutant allele frequency (Fig. 2c). After that, frameshift and stop gained/lost were the next most common. 
a Prevalence of WD from epidemiological studies

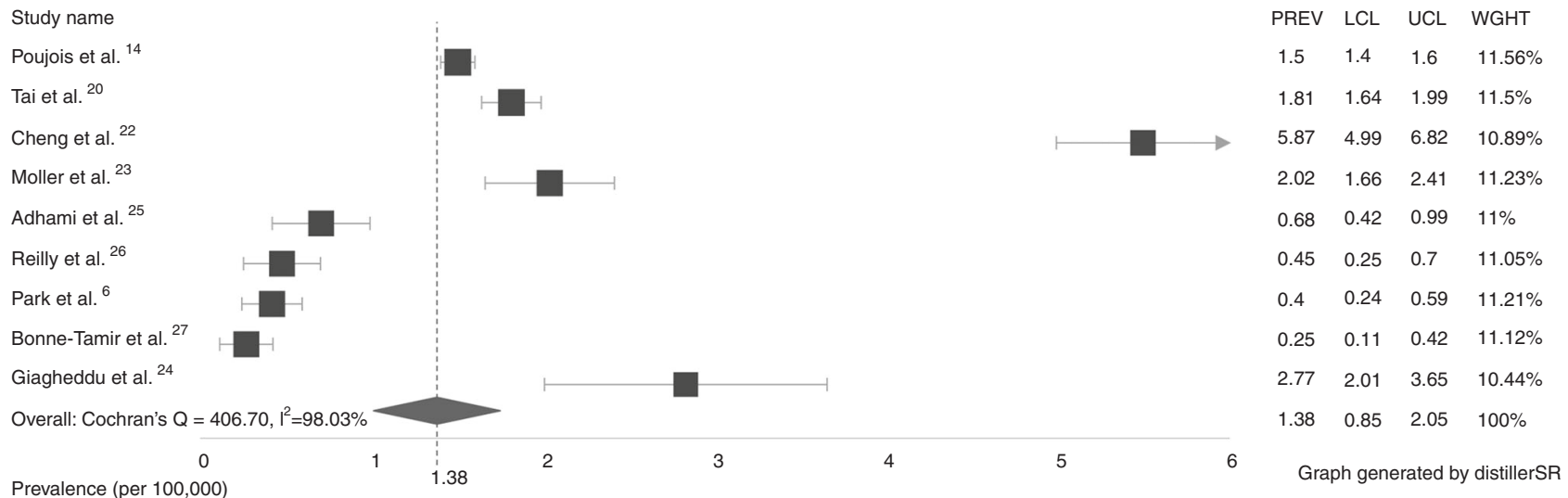

b

WD mutant allele frequency from genetic studies

Study name

Jang et al. ${ }^{7}$

Jang et al. ${ }^{7}$ (2)

kim at al. ${ }^{30}$

Coffey et al. ${ }^{3}$

Zappu et al. ${ }^{31}$

Zappu et al. ${ }^{31}$ (2)

Gialluisi et al. ${ }^{17}$

Mak et al. ${ }^{32}$

Krumina et al. ${ }^{15}$

Olivarez et al. ${ }^{8}$

Overall:Cochran's $Q=39.03 ; I^{2}=76.94$

0

Mutant allele frequency $(\%)$

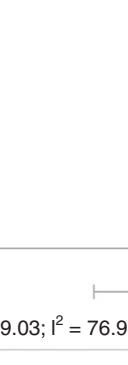

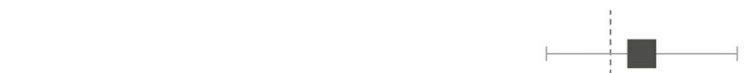

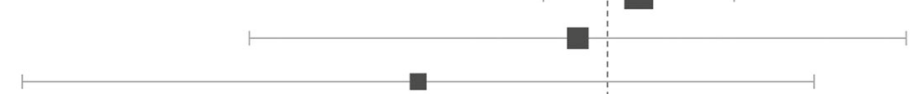

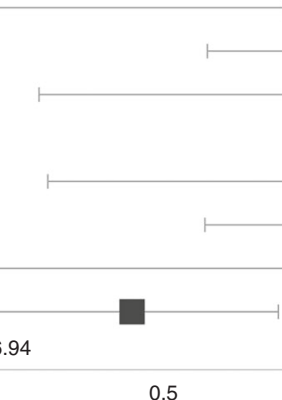

0.5

Fig. 1 Forest plots of previous studies of Wilson disease (WD) prevalence. (a) Prevalence estimates derived from epidemiological studies. (b) Mutant allele frequencies derived from genetic studies. Error bars represent 95\% confidence intervals (CIs). Overall estimates are calculated using random effects models. LCL lower 95\% confidence interval, MAF mutant allele frequency, PREV prevalence, UCL upper 95\% confidence interval, WGHT weight.

Fifty percent of the total allele frequency was accounted for by the nine most frequent variants, the top five of which are listed in Table 3. The most frequently previously reported variant in the European population, p.His1069Gln, ranks third among these.

\section{Predicted pathogenic variants}

To estimate the true genetic prevalence of $\mathrm{WD}$, including pathogenic variants that have not yet been identified in patients, we examined all variants reported in humans that caused major functional or structural changes (frameshift, premature stop codon, splice donor, and splice acceptor variants). After frequency filtering, 113/11,520 (0.98\%) variants met these criteria and were found in the gnomAD data set. Of these, 60 had not been previously identified in WD patients. When combined with the reported pathogenic variants above, the global mutant allele frequency of WD was 0.0124 , equating to a birth prevalence of 15.4 per 100,000 (95\% CI: 14.4-16.5), or 1 per 6494 (Fig. 2b and
Table S1). The East Asian population had the highest prevalence of 29.7 per 100,000 (95\% CI: $23.8-37.0$ ) and the Finnish population had the lowest prevalence at 2.37 per 100,000 (95\% CI 1.7-3.4).

\section{DISCUSSION}

In this study, we have produced an unbiased description of the genetic prevalence of Wilson disease, both globally and across seven major ethnicities, and this was found to be higher than previous estimates. In addition, we have collated a publicly available, up-to-date database of $A T P 7 B$ gene variants with robust classifications of pathogenicity.

Previous estimates of WD prevalence are extremely heterogeneous, which may be accounted for by differences in population, diagnosis, and methodology. In particular, it should be remembered that the prevalence of WD in isolated populations, such as the Canary Islands and Crete, may be over 35 per 100,000 . In contrast, using the large and diverse sample represented by the gnomAD data set (comprising 123,136 
a

(i) Reported disease variants

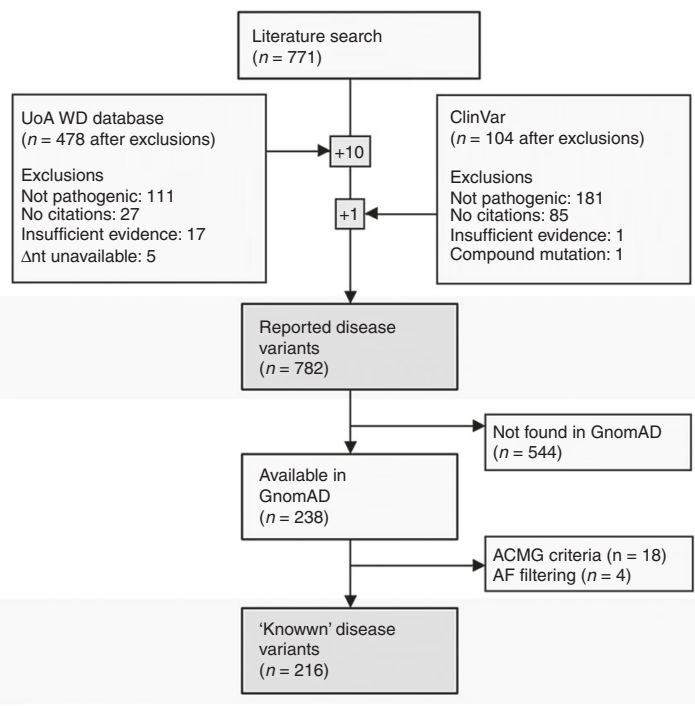

(ii) Predicted disease variants

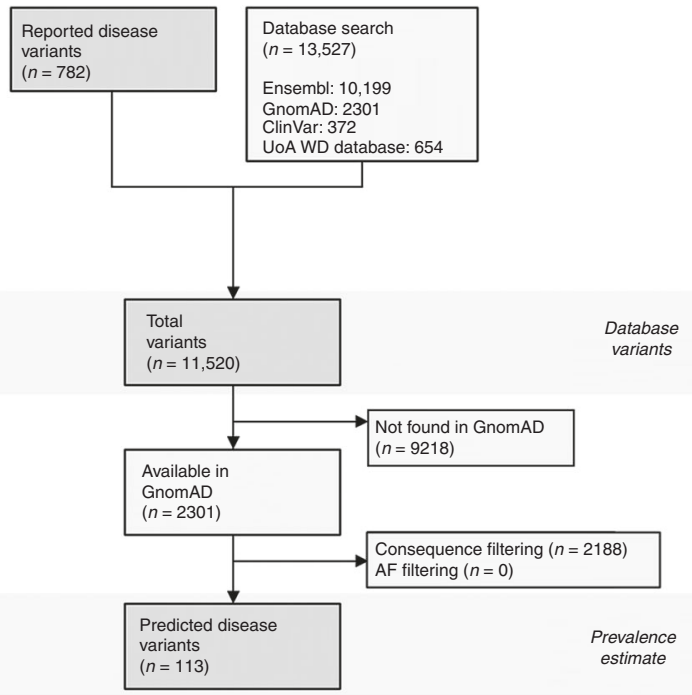

b

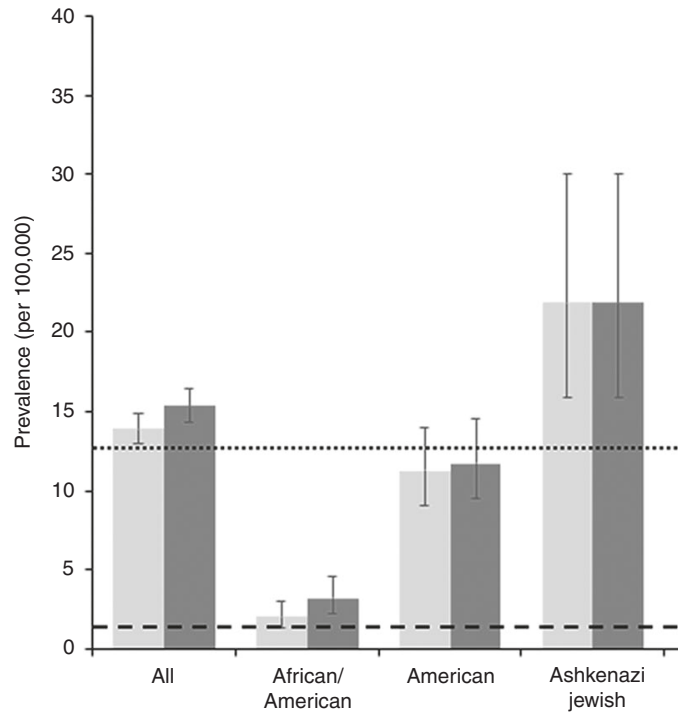

C

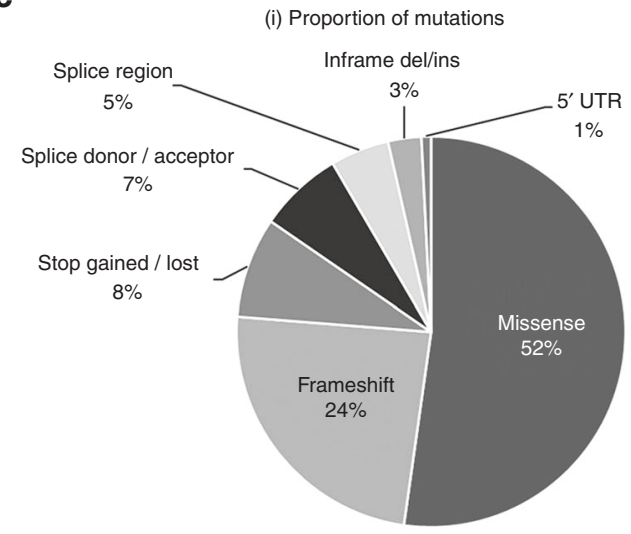

(ii) Proportion of total prevalence

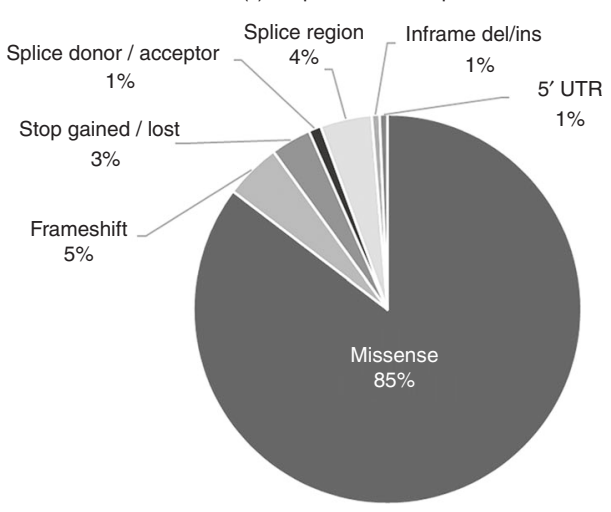


Fig. 2 ATP7B gene variants and gnomAD allele frequencies. (a) Identification and classification of $A T P 7 B$ disease variants. $A T P 7 B$ variants were compiled into a new, comprehensive, publicly available database. Additionally, these were filtered to obtain estimates of the prevalence of Wilson disease (WD). (i) Reported disease variants were identified by a systematic search of the literature and cross-referencing with existing databases. Variants found in the gnomAD data set were then filtered by frequency and American College of Medical Genetics and Genomics (ACMG) criteria to give "known disease variants." (ii) Reported disease variants and variants from existing databases were combined to give a list of all known ATP7B variants. Those found in the gnomAD data set were filtered by frequency and mutation consequence-variants causing major functional or structural changes (frameshift, premature stop codon, splice donor, and splice acceptor variants) were classified as "predicted disease variants." $A F$ allele frequencies, $n=$ number of variants, $\Delta$ nt $=$ nucleotide change, UoA University of Alberta. (b) WD disease prevalence estimated from gnomAD allele frequencies. Estimates of WD prevalence for seven ethnicities were calculated using the gnomAD allele frequencies of known and predicted disease variants (identified as per a). For comparison, estimates of prevalence from the meta-analyses of epidemiological and genetic studies are shown by the red and the yellow dotted lines, respectively. Error bars represent 95\% confidence intervals (Cls) calculated using the Wilson score method. (c) Mutation consequences of "known disease variants." (i) Pie chart of the number of variants resulting in each mutation consequence. (ii) Pie chart of the proportion of the total mutant allele frequency accounted for by each mutation consequence. del deletion, ins insertion, UTR untranslated region.

Table 3 Characteristics of the five most frequent known disease variants

\begin{tabular}{lllllcc} 
Genomic location & cDNA change & Protein change & SIFT & PolyPhen & GnomAD AF & References \\
\hline 51946372 & c. 2972 C $>$ T & p.Thr991Met & Deleterious (0) & Probably_damaging (0.999) & 0.00126 & 33,34 \\
51935019 & c. $4135 C>T$ & p.Pro1379Ser & Deleterious (0) & Probably_damaging (0.978) & 0.00106 & 33 \\
51944145 & c. $3207 C>A$ & p.His1069Gln & Deleterious (0) & Probably_damaging (1) & 0.00101 & $35-37$ etc. \\
51950132 & c. $2605 G>A$ & p.Gly869Arg & Deleterious (0) & Probably_damaging (0.996) & 0.00072 & $28,34,38$ etc. \\
51961849 & c. $1934 T>$ G & p.Met645Arg & Tolerated (0.42) & Benign (0) & 0.00048 & $38-40$ etc. \\
\hline
\end{tabular}

$A F$ allele frequency, CDNA complementary DNA.

exome sequences and 15,496 genome sequences), we deduce that the global genetic prevalence of WD at birth is approximately 13.9 to 15.4 per 100,000 . We have highlighted that patients of East Asian origin are at the greatest risk of WD, whereas those of Finnish origin have the lowest genetic prevalence.

In particular, our estimate of 13.9 per 100,000 , derived from known pathogenic variants, is very similar to the prevalence from the meta-analysis of genetic studies (12.7 per 100,000), but both of these estimates are significantly higher than the prevalence from the meta-analysis of epidemiological studies (1.38 per 100,000$)$. There are several possible reasons for this disparity.

Firstly, the genetic prevalence calculated in this study does not account for the incomplete penetrance of variants, as penetrance data are lacking. If the difference between epidemiological (1.4 per 100,000) and genetic (13.9 per $100,000)$ estimates were due to incomplete penetrance alone then the overall disease penetrance would be $10 \%$. However, the genetic studies contributing to the meta-analysis were mostly based on a maximum of six common pathogenic variants, with the exception of Coffey et al. ${ }^{3}$ and Gialluisi et al. ${ }^{17}$ Because these are mostly well-reported variants, which have repeatedly been screened for in control populations, it is unlikely that any of these could have a penetrance low enough to account for the extent of disparity between genetic and epidemiological estimates.

Methodological differences may also contribute. WD commonly presents during the second and third decades of life, whereas the genotype is present from birth. Thus, the clinical phenotype quantified by epidemiological studies only exists for about $80 \%$ of an average 70 -year lifespan.
Finally, this disparity may be evidence of underdiagnosis of WD on a population level, as has been repeatedly previously suggested, ${ }^{3,7}$ or of delayed diagnosis and consequent early deaths. With good compliance, the treatment for WD is highly effective and diagnostic failure has been reported to be the principal cause of death in WD patients. ${ }^{18}$ Underdiagnosis may be due to milder disease phenotypes, or single-organ system phenotypes, or application of diagnostic criteria recently recognized as inappropriately narrow. ${ }^{1}$

Thus, we have demonstrated the power and potential limitations of using rapidly expanding genomic databases such as gnom $A D$ to estimate the prevalence of recessive diseases. These conclusions are, however, limited by the assumptions of the Hardy-Weinberg equation and many of the consequence-predicted variants are not yet supported by in vitro data. Moreover, pathogenic classification of our previously reported variants is reliant upon accurate diagnosis by the papers we screened.

We also implemented the method for filtering variants by frequency proposed by Whiffin et al. ${ }^{13}$ As the authors note, a limitation of this procedure is the difficulty in obtaining reliable estimates of penetrance and maximum allelic contribution. Therefore, an upper bound for the maximum credible allele frequency was used here to avoid filtering out potentially pathogenic variants. Although not optimal, such a method is still more stringent than the standard practice of discarding variants more frequent than the arbitrary MAF of $5 \%$. It should also be noted that the use of frequency filtering was only intended to remove variants too common to realistically be pathogenic, rather than to define pathogenic variants. Indeed, only four variants were filtered out here and were we to assume $100 \%$ 
penetrance in this formula, only one additional variant would be filtered, giving a prevalence estimate of 11.6 per 100,000 .

Our finding of 782 reported disease variants and 60 predicted pathogenic variants associated with WD is an update on previous lists of variants and highlights the mutational spectrum of the disease. The most common pathogenic variants are missense pathogenic variants (accounting for $85 \%$ of the prevalence), but large deletions and insertions have also been reported. This should be considered in efforts to develop an effective screening program or in targeted genetic testing for the disease.

In summary, the genetic prevalence of WD is much higher than epidemiological estimates, potentially indicating underdiagnosis or the existence of less severe phenotypes. We have also produced more reliable global and ethnicity-specific estimates for WD genetic prevalence and in the process, a new up-to-date database of WD variants. These results provide important baseline data for clinical use, genetic counseling, and informing future research in $A T P 7 B$.

\section{ELECTRONIC SUPPLEMENTARY MATERIAL}

The online version of this article (https://doi.org/10.1038/s41436018-0309-9) contains supplementary material, which is available to authorized users.

\section{ACKNOWLEDGEMENTS}

The authors would like to thank Patrick McKeirnan (Pittsburgh, PA, USA) for his advice during this project. We are also grateful to Diane Cox, Georgina Maclntyre, and the whole team from the University of Alberta Wilson Disease Mutation Database for their support in this work.

\section{DISCLOSURE}

The authors declare no conflicts of interest.

\section{REFERENCES}

1. European Association for Study of Liver. EASL clinical practice guidelines: Wilson's disease. J Hepatol. 2012;56:671-685.

2. Lovicu M, Dessì V, Lepori MB, et al. The canine copper toxicosis gene MURR1 is not implicated in the pathogenesis of Wilson disease. J Gastroenterol. 2006;41:582-587.

3. Coffey AJ, Durkie M, Hague S, et al. A genetic study of Wilson's disease in the United Kingdom. Brain J Neurol. 2013;136 Pt 5:1476-1487.

4. Bugbee D, Davies L, Kenney S, Cox DW. Wilson Disease Mutation Database. http://www.wilsondisease.med.ualberta.ca/database.asp. Accessed 15 March 2018.

5. Scheinberg I, Sternlieb I. Wilson's disease. Philadelphia: WB Saunders; 1984.

6. Park RH, McCabe P, Fell GS, Russell RI. Wilson's disease in Scotland. Gut. 1991;32:1541-1545.

7. Jang J-H, Lee T, Bang S, Kim Y-E, Cho E-H. Carrier frequency of Wilson's disease in the Korean population: a DNA-based approach. J Hum Genet. 2017;62:815-818. https://doi.org/10.1038/jhg.2017.49

8. Olivarez L, Caggana M, Pass KA, Ferguson P, Brewer GJ. Estimate of the frequency of Wilson's disease in the US Caucasian population: a mutation analysis approach. Ann Hum Genet. 2001;65 Pt 5:459-463.

9. Wallace DF, Subramaniam VN. The global prevalence of HFE and nonHFE hemochromatosis estimated from analysis of next-generation sequencing data. Genet Med. 2016;18:618-626.
10. Downes MJ, Brennan ML, Williams HC, Dean RS. Development of a critical appraisal tool to assess the quality of cross-sectional studies (AXIS). BMJ Open. 2016;6:e011458.

11. Deeks JJ, Altman DG, Bradburn MJ. Statistical methods for examining heterogeneity and combining results from several studies in metaanalysis. In: Systematic reviews in health care: meta-analysis in context. (Egger M, Smith GD, Altman DG, eds.) p. 285-312, London: BMJ Publishing Group; 2008.

12. Kenney SM, Cox DW. Sequence variation database for the Wilson disease copper transporter, ATP7B. Hum Mutat. 2007;28:1171-1177.

13. Whiffin N, Minikel $E$, Walsh $R$, et al. Using high-resolution variant frequencies to empower clinical genome interpretation. Genet Med. 2017;19:1151-1158.

14. Ferenci $P$. Regional distribution of mutations of the ATP7B gene in patients with Wilson disease: impact on genetic testing. Hum Genet. 2006;120:151-159.

15. Krumina A, Keiss J, Sondore V, et al. From clinical and biochemical to molecular genetic diagnosis of Wilson disease in Latvia. Genetika. 2008; 44:1379-1384.

16. Richards S, Aziz N, Bale S, et al. Standards and guidelines for the interpretation of sequence variants: a joint consensus recommendation of the American College of Medical Genetics and Genomics and the Association for Molecular Pathology. Genet Med. 2015;17:405-424.

17. Gialluisi A, Incollu S, Pippucci $\mathrm{T}$, et al. The homozygosity index $(\mathrm{HI})$ approach reveals high allele frequency for Wilson disease in the Sardinian population. Eur J Hum Genet. 2013;21:1308-1311.

18. Walshe JM. Cause of death in Wilson disease. Mov Disord. 2007;22: 2216-2220.

19. Poujois A, Woimant F, Samson S, Chaine P, Girardot-Tinant N, Tuppin P Characteristics and prevalence of Wilson's disease: a 2013 observational population-based study in France. Clin Res Hepatol Gastroenterol. 2017;42:57-63. https://doi.org/10.1016/j.clinre.2017.05.011

20. Tai C-S, Wu J-F, Chen H-L, Hsu H-Y, Chang M-H, Ni Y-H Modality of treatment and potential outcome of Wilson disease in Taiwan: a population-based longitudinal study. J Formos Med Assoc Taiwan Yi Zhi. 2017;117:421-426. https://doi.org/10.1016/j.jfma.2017.05.008

21. Lai C-H, Tseng H-F. Population-based epidemiologic study of Wilson's disease in Taiwan. Eur J Neurol. 2010;17:830-833.

22. Cheng $\mathrm{N}$, Wang $\mathrm{K}, \mathrm{Hu}$ W, et al. Wilson disease in the South Chinese Han population. Can J Neurol Sci. 2014;41:363-367.

23. Moller LB, Horn N, Jeppesen TD, et al. Clinical presentation and mutations in Danish patients with Wilson disease. Eur J Hum Genet. 2011;19:935-941.

24. Giagheddu A, Demelia L, Puggioni G, et al. Epidemiologic study of hepatolenticular degeneration (Wilson's disease) in Sardinia (1902-1983). Acta Neurol Scand. 1985;72:43-55.

25. Adhami EJ, Cullufi P. Wilson's disease in Albania. Panminerva Med. 1995; 37:18-21.

26. Reilly M, Daly L, Hutchinson M. An epidemiological study of Wilson's disease in the Republic of Ireland. J Neurol Neurosurg Psychiatry. 1993;56:298-300.

27. Bonne-Tamir B, Frydman M, Agger MS, et al. Wilson's disease in Israel: a genetic and epidemiological study. Ann Hum Genet. 1990;54 Pt 2:155-168.

28. García-Villarreal L, Daniels S, Shaw SH, et al. High prevalence of the very rare Wilson disease gene mutation Leu708Pro in the Island of Gran Canaria (Canary Islands, Spain): a genetic and clinical study. Hepatol. 2000;32:1329-1336.

29. Dedoussis GVZ, Genschel J, Sialvera T-E, et al. Wilson disease: high prevalence in a mountainous area of Crete. Ann Hum Genet. 2005;69 Pt 3:268-274.

30. Kim G-H, Yang JY, Park J-Y, Lee JJ, Kim JH, Yoo H-W. Estimation of Wilson's disease incidence and carrier frequency in the Korean population by screening ATP7B major mutations in newborn filter papers using the SYBR green intercalator method based on the amplification refractory mutation system. Genet Test. 2008;12: 395-399.

31. Zappu A, Magli O, Lepori MB, et al. High incidence and allelic homogeneity of Wilson disease in 2 isolated populations: a prerequisite for efficient disease prevention programs. J Pediatr Gastroenterol Nutr. 2008;47:334-338

32. Mak CM, Lam C-W, Tam S, et al. Mutational analysis of 65 Wilson disease patients in Hong Kong Chinese: identification of 17 novel mutations and its genetic heterogeneity. J Hum Genet. 2008;53:55-63. 
33. Cox DW, Prat L, Walshe JM, Heathcote J, Gaffney D. Twenty-four novel mutations in Wilson disease patients of predominantly European ancestry. Hum Mutat. 2005;26:280.

34. Lepori MB, Lovicu M, Dessi V, et al. Twenty-four novel mutations in Wilson disease patients of predominantly Italian origin. Genet Test. 2007;11:328-332.

35. Tanzi RE, Petrukhin K, Chernov I, et al. The Wilson disease gene is a copper transporting ATPase with homology to the Menkes disease gene. Nat Genet. 1993;5:344-350.

36. Zali N, Mohebbi SR, Esteghamat S, et al. Prevalence of ATP7B gene mutations in Iranian patients with Wilson disease. Hepat Mon. 2011:11:890-894.
37. Figus $A$, Angius $A$, Loudianos $G$, et al. Molecular pathology and haplotype analysis of Wilson disease in Mediterranean populations. Am J Hum Genet. 1995:57:1318-1324.

38. Shah $A B$, Chernov I, Zhang HT, et al. Identification and analysis of mutations in the Wilson disease gene (ATP7B): population frequencies, genotype-phenotype correlation, and functional analyses. Am J Hum Genet. 1997;61:317-328.

39. Kalinsky H, Funes A, Zeldin A, et al. Novel ATP7B mutations causing Wilson disease in several Israeli ethnic groups. Hum Mutat. 1998;11:145-151.

40. Loudianos G, Dessì V, Lovicu $M$, et al. Further delineation of the molecular pathology of Wilson disease in the Mediterranean population. Hum Mutat. 1998:12:89-94. 